PROCEEDINGS OF THE

AMERICAN MATHEMATICAL SOCIETY

Volume 125, Number 2, February 1997, Pages 323-327

S 0002-9939(97)03539-9

\title{
GROUPS WITH MANY NORMAL-BY-FINITE SUBGROUPS
}

\author{
SILVANA FRANCIOSI AND FRANCESCO DE GIOVANNI
}

(Communicated by Ronald M. Solomon)

\begin{abstract}
A subgroup $H$ of a group $G$ is said to be normal-by-finite if the core $H_{G}$ of $H$ in $G$ has finite index in $H$. In this article groups satisfying the minimal condition on subgroups which are not normal-by-finite and groups with finitely many conjugacy classes of subgroups which are not normal-byfinite are characterized.
\end{abstract}

\section{INTRODUCTION}

Let $\chi$ be a property pertaining to subgroups. Many authors have investigated the structure of groups in which only few subgroups do not have the property $\chi$, for various natural choices of $\chi$. Here the affirmation that the property $\chi$ does not hold only for a few subgroups of the group can of course be interpreted in several different ways, especially when the groups considered are infinite. A natural interpretation is that of asking that the group satisfies the minimal condition on subgroups which do not have the property $\chi$. This point of view was adopted in [7], and more recently in [4] and [5]. In particular, the authors investigated in [5] the structure of groups satisfying the minimal condition on subgroups having infinite index in their normal closure. In the first part of this article we will consider groups satisfying the minimal condition on subgroups which are not normal-by-finite. Here a subgroup $H$ of a group $G$ is called normal-by-finite if the core $H_{G}$ of $H$ in $G$ has finite index in $H$. Groups in which all subgroups are normal-by-finite have been considered in [3] and [10], where in particular it is proved that if $G$ is a group with that property, and all periodic homomorphic images of $G$ are locally finite, then $G$ is abelian-byfinite. Moreover, the structure of groups in which every subnormal subgroup is normal-by-finite has been studied in [6]. Here we prove the following theorem.

Theorem A. Let $G$ be a group whose periodic homomorphic images are locally finite. If $G$ satisfies the minimal condition on subgroups which are not normal-byfinite, then either $G$ is a Černikov group or all its subgroups are normal-by-finite.

The above theorem, together with the quoted results on groups in which all subgroups are normal-by-finite, has the following obvious consequence.

Corollary. Let $G$ be a group whose periodic homomorphic images are locally finite. If $G$ satisfies the minimal condition on subgroups which are not normal-by-finite, then $G$ is abelian-by-finite.

Received by the editors May 11, 1995.

1991 Mathematics Subject Classification. Primary 20F22.

(C)1997 American Mathematical Society 
The minimal condition on subgroups which do not have a prescribed property is closely related to the imposition that there exist only finitely many conjugacy classes of subgroups which do not have the same property. Groups with a condition of this type have recently been considered in [2], [4], [5], [9]. We obtain here the following result on groups with finitely many conjugacy classes of subgroups which are not normal-by-finite.

Theorem B. Let $G$ be a group whose periodic homomorphic images are locally finite. If $G$ has finitely many conjugacy classes of subgroups which are not normalby-finite, then every subgroup of $G$ is normal-by-finite.

Most of our notation is standard and can for instance be found in [8].

\section{Proofs}

Proof of Theorem A. Let $G$ be a group satisfying the minimal condition on subgroups which are not normal-by-finite, and suppose also that every periodic homomorphic image of $G$ is locally finite. Let $x$ be any element of infinite order of $G$. Then there exists a positive integer $n$ such that $\left\langle x^{n}\right\rangle$ is a normal-by-finite subgroup of $G$. As $\left\langle x^{n}\right\rangle$ has finite index in $\langle x\rangle$, we have that also $\langle x\rangle$ is normal-by-finite in $G$. Clearly the subgroup $X$ generated by all infinite cyclic normal subgroups of $G$ is abelian, and the above remark shows that the factor group $G / X$ is periodic, and hence locally finite. Thus every periodic section of $G$ is locally finite. Assume that the set $\mathfrak{L}$ of all subgroups of $G$ which are not normal-by-finite is not empty, and let $L$ be a minimal element of $\mathfrak{L}$. Then every proper subgroup $H$ of $L$ is normalby-finite in $G$, so that in particular $H / H_{L}$ is finite, and so all subgroups of $L$ are normal-by-finite. It follows that $L$ is abelian-by-finite (see [10], Theorem 2). On the other hand, $L$ does not contain proper subgroups of finite index, so that it is a radicable abelian group. Clearly $L$ cannot be generated by two proper subgroups, and hence it is a subgroup of type $p^{\infty}$ for some prime $p$. Suppose first that $G$ is not periodic, so that it follows from the first part of the proof that $G$ contains an infinite cyclic normal subgroup $\langle a\rangle$. Since $\langle a\rangle \cap L=1$, the chain

$$
\langle a, L\rangle>\left\langle a^{2}, L\right\rangle>\left\langle a^{4}, L\right\rangle>\cdots>\left\langle a^{2^{n}}, L\right\rangle>\cdots
$$

is strictly descending, and hence there exists in $\langle a\rangle$ an element $b \neq 1$ such that the subgroup $K=\langle b, L\rangle$ is normal-by-finite in $G$. Then $L$ is contained in the core $K_{G}$ of $K$ in $G$, so that $K=K_{G}$ is a normal subgroup of $G$. On the other hand, $K=\langle b\rangle \times L$ and so $L$ is the subgroup of all elements of finite order of $K$. Thus $L$ is normal in $G$, and this contradiction shows that if $G$ is not periodic, then all subgroups of $G$ are normal-by-finite. Suppose now that $G$ is periodic, and hence locally finite. If $G$ is not a Černikov group, by a result of Šunkov [11] it does not satisfy the minimal condition on abelian subgroups. Thus $G$ contains an abelian subgroup $A$ which is a direct product of infinitely many subgroups of prime order. Clearly $A$ can be chosen in such a way that $A \cap L=1$. Since $A$ does not contain Prüfer subgroups, it follows from the first part of the proof that every subgroup of $A$ is normal-by-finite in $G$. Therefore there exists in $A$ a chain of $G$-invariant subgroups

$$
A_{1}>A_{2}>\cdots>A_{n}>\cdots
$$

such that every $A_{n}$ has finite index in $A$. As $A \cap L=1$, the subgroup $L A_{n}$ properly contains $L A_{n+1}$ for each $n$, and so there exists a positive integer $m$ such that $L A_{m}$ 
is a normal-by-finite subgroup of $G$. But $L$ is a group of type $p^{\infty}$, so that $L$ is contained in the core of $L A_{m}$ in $G$, and hence the subgroup $L A_{m}$ is normal in $G$. In particular, $L^{G}$ is contained in $L A_{m}$, and hence $L^{G}=L\left(A_{m} \cap L^{G}\right)$. Since $L$ is not characteristic in $L^{G}$, the intersection $B=A_{m} \cap L^{G}$ must be infinite. As above, it can be shown that $B$ contains a proper $G$-invariant subgroup of finite index $C$ such that $L C$ is normal in $G$. Clearly $B \leq L^{G} \leq L C$, so that $B=B \cap L C=C$. This contradiction proves that, if $G$ is not a Cernikov group, then every subgroup of $G$ is normal-by-finite.

Groups satisfying the minimal condition on subgroups with a given property $\chi$ are naturally involved in the investigations concerning groups with a finite number of conjugacy classes of $\chi$-subgroups. This is a consequence of the following result of Zaicev.

Lemma 1 (see [1], Lemma 4.6.3). Let $G$ be a group locally satisfying the maximal condition on subgroups. If $H$ is a subgroup of $G$ such that $H^{x} \leq H$ for some element $x$ of $G$, then $H^{x}=H$.

Lemma 2. Let $G$ be a Černikov group with finitely many conjugacy classes of subgroups which are not normal-by-finite. Then every subgroup of $G$ is normal-byfinite.

Proof. Assume first that $G$ contains a non-normal subgroup $P$ of type $p^{\infty}$ for some prime $p$. Then there exists in $G$ another subgroup $Q$ of type $p^{\infty}$ such that $\langle P, Q\rangle=P \times Q$. For every non-negative integer $n$ let $Q_{n}$ be the subgroup of order $p^{n}$ of $Q$. Then the subgroups $P Q_{n}$ are pairwise non-isomorphic, and so there exists $n$ such that $H=P Q_{n}$ is normal-by-finite in $G$. Clearly $P$ is a characteristic subgroup of the core $H_{G}$ of $H$, and so is normal in $G$. This contradiction shows that every Prüfer subgroup of $G$ is normal. Let $X$ be any subgroup of $G$. Then the finite residual $Y$ of $X$ is normal in $G$, and so $X$ is a normal-by-finite subgroup of $G$.

Lemma 3. Let $G$ be a group whose periodic homomorphic images are locally finite. If $G$ has finitely many conjugacy classes of subgroups which are not normal-byfinite and every cyclic subgroup of $G$ is normal-by-finite, then all subgroups of $G$ are normal-by-finite.

Proof. Let $X$ be the subgroup generated by all infinite cyclic normal subgroups of $G$. Then $X$ is abelian and the factor group $G / X$ is periodic, since every cyclic subgroup of $G$ is normal-by-finite. It follows that $G / X$ is locally finite, and $G$ locally satisfies the maximal condition on subgroups. Thus $G$ satisfies the minimal condition on subgroups which are not normal-by-finite by Lemma 1, and hence by Theorem A we have that either $G$ is a Černikov group or all its subgroups are normal-by-finite. On the other hand, if $G$ is a Cernikov group every subgroup of $G$ is normal-by-finite by Lemma 2, and so the lemma is proved.

Proof of Theorem B. Assume first that $G$ is periodic. Then $G$ is locally finite, and by Lemma 1 it satisfies the minimal condition on subgroups which are not normal-by-finite. Application of Theorem A and Lemma 2 yields that in this case all subgroups of $G$ are normal-by-finite. Suppose now that $G$ is not periodic, and assume by contradiction that $G$ contains subgroups which are not normal-by-finite. Then by Lemma 3 there exists an element of infinite order $x$ of $G$ such that the subgroup $\langle x\rangle$ has trivial core in $G$. Let $p$ be any prime. Then there exist positive 
integers $r$ and $s$ such that $r>s$ and the subgroups $\left\langle x^{p^{r}}\right\rangle$ and $\left\langle x^{p^{s}}\right\rangle$ are conjugate. Let $g$ be an element of $G$ such that $\left\langle x^{p^{r}}\right\rangle^{g}=\left\langle x^{p^{s}}\right\rangle$, and put $y=x^{p^{s}}$ and $k=r-s$. Thus

$$
\left(y^{g}\right)^{p^{k}}=\left(y^{p^{k}}\right)^{g}=\left(x^{p^{r}}\right)^{g}=y^{ \pm 1}
$$

so that

$$
\langle y\rangle<\left\langle y^{g}\right\rangle<\left\langle y^{g^{2}}\right\rangle<\cdots
$$

and $Y_{p}=\bigcup_{n \geq 0}\left\langle y^{g^{n}}\right\rangle$ is a subgroup of $G$ isomorphic with the additive group of rational numbers whose denominators are powers of $p$. In particular, if $p_{1}$ and $p_{2}$ are distinct primes, the subgroups $Y_{p_{1}}$ and $Y_{p_{2}}$ are not conjugate, and hence there exists a prime $q$ such that $Y_{q}$ is normal-by-finite in $G$. Thus the core $A$ of $Y_{q}$ in $G$ is a torsion-free abelian normal subgroup of rank 1 of $G$, and $A \cap\langle x\rangle \neq 1$.

Let $B$ be a cyclic non-trivial subgroup of $A$, and for every prime $p$ let $A(p) / B$ be the $p^{\prime}$-component of $A / B$, so that $A / A(p)$ is a $p$-group. If $p$ and $q$ are distinct primes such that the subgroups $A(p)$ and $A(q)$ are not normal-by-finite in $G$, then $A(p)$ and $A(q)$ are both properly contained in $A$, and hence they are not conjugate. Since $G$ has finitely many conjugacy classes of subgroups which are not normal-byfinite, it follows that all but finitely many $A(p)$ are normal-by-finite. Let $k$ be the number of conjugacy classes of subgroups of $G$ which are not normal-by-finite, and let $\pi=\left\{p_{1}, \ldots, p_{k+2}\right\}$ be a set consisting of $k+2$ distinct primes such that the subgroups $A\left(p_{1}\right), \ldots, A\left(p_{k+2}\right)$ are normal-by-finite in $G$. Then also the subgroup $A_{0}=\bigcap_{i=1}^{k+2} A\left(p_{i}\right)$ is normal-by-finite in $G$, so that the core $X$ of $A_{0}$ in $G$ has finite index in $A_{0}$. Clearly $X \cap B \neq 1$, and hence replacing $B$ by $X \cap B$ it can be assumed without loss of generality that $B$ is contained in $X$. The factor group $X / B$ is a $\pi^{\prime}$-group, and hence $X^{p_{i}} \cap B=B^{p_{i}}$ for every $i \leq k+2$. Assume that the subgroups $B^{p_{i}}$ and $B^{p_{j}}$ are conjugate. Then $B^{p_{j}} \leq X^{p_{i}} \cap B=B^{p_{i}}$, so that $p_{i}=p_{j}$ and $i=j$. It follows that the subgroups $B^{p_{1}}, \ldots, B^{p_{k+2}}$ are pairwise non-conjugate, and hence at least two of them are normal-by-finite in $G$. Therefore $B$ is normal-by-finite in $G$. On the other hand, $B \cap\langle x\rangle \neq 1$ and hence also $\langle x\rangle$ is normal-by-finite in $G$. This contradiction proves that all subgroups of $G$ are normal-by-finite.

\section{REFERENCES}

[1] B. Amberg, S. Franciosi and F. de Giovanni, Products of Groups, Oxford Mathematical Monographs, Clarendon Press, Oxford, 1992. MR 94h:20001

[2] R. Brandl, S. Franciosi and F. de Giovanni, Groups with finitely many conjugacy classes of non-normal subgroups, Proc. Roy. Irish Acad. Sect. A 95 (1995), 17-27. CMP 1996:6

[3] J. Buckley, J. C. Lennox, B. H. Neumann, H. Smith, and J. Wiegold, Groups with all subgroups normal-by-finite, J. Austral. Math. Soc. Ser. A 59 (1995), 384-398. MR 96h:20076

[4] S. Franciosi and F. de Giovanni, Groups satisfying the minimal condition of non-subnormal subgroups, Proceedings of "Infinite Groups 1994", de Gruyter, Berlin, 63-72.

[5] S. Franciosi and F. de Giovanni, Groups satisfying the minimal condition on certain nonnormal subgroups, Proceedings of "Groups-Korea 1994", de Gruyter, Berlin, 107-118.

[6] S. Franciosi, F. de Giovanni, and M. L. Newell, Groups whose subnormal subgroups are normal-by-finite, Comm. Algebra 23 (1995), 5483-5497. MR 96h:20058

[7] R. E. Phillips and J. S. Wilson, On certain minimal conditions for infinite groups, J. Algebra $\mathbf{5 1}$ (1978), 41-68. MR 58:11126

[8] D. J. S. Robinson, Finiteness Conditions and Generalized Soluble Groups. Parts I, II, Springer, Berlin, 1972. MR 48:111314,111315

[9] H. Smith, Groups with finitely many conjugacy classes of subgroups with large subnormal defect, Glasgow Math. J. 37 (1995), 69-71. MR 96a:22048 
[10] H. Smith and J. Wiegold, Locally graded groups with all subgroups normal-by-finite, J. Austral. Math. Soc. Ser. A 60 (1996), 222-227. CMP 1996:8

[11] V. P. Šunkov, On the minimality problem for locally finite groups, Algebra and Logic 9 (1970), 137-151. MR 44:295 (Russian original)

Dipartimento di Matematica e Applicazioni, Università di Napoli "Federico II", ComPlesso Universitario Monte S. Angelo, Via Cintia, I 80126 Napoli, Italy

E-mail address: degiova@matna2.dma.unina.it 